\title{
Rare Cause of Acute Tetraplegia and Respiratory Arrest: Cervicomedullary Neuroenteric Cyst in a Child: Case Report
}

\author{
Hakan Ozalp, ${ }^{1}$ Vural Hamzaoglu, ${ }^{1}$ Derya Karatas, ${ }_{1}^{1}$ Ahmet Dagtekin, ${ }^{1}$ Mehmet Yildız, ${ }^{2}$ and Emel Avcı ${ }^{1}$
}

\begin{abstract}
Background: Neuroenteric cysts (NCs) are benign, rare congenital lesions which are most commonly found in the lower cervical and upper thoracic segments. Even though these are benign lesions, it may present with fatal neurologic deterioration particularly in pediatric patients. Case Report: We here report a 16-year-old boy who has unique manifestation with acute tetraplegia and respiratory arrest in emergency room. According to English literature, this is the first case of cervicomedullary intradural neuroenteric cyst which causes acute tetraplegia and respiratory arrest in a child. Conclusion: The NCs can be manifested with unpredictable, life threating clinical presentation. The exact pathophysiology of an acute neurological deterioration is still unclear, however prolonged latent period with minor symptom should be considered carefully in child population.
\end{abstract}

Keywords: neuroenteric cysts, cervicomedullary junction, clinical course, child patient

\section{Introduction}

Neuroenteric cysts (NCs) also known as enterogenous or endodermal cysts are rare, benign congenital lesions and represent around $0.01 \%$ of central nervous system tumors. ${ }^{1,2)}$ These lesions are usually located in the intradurally and extramedullary most commonly seen in the lower cervical, upper thoracic region. ${ }^{3-5)}$

The precise etiology of NCs are still uncertain. The various theories have been written to explain the embryogenesis of NCs. The first theory is as follows: In the normal development of human embryogenesis, a trilaminar germ disk consisting of ectoderm, mesoderm and endoderm is formed at the 3rd week of development. After the mesodermal cells lying between the amniotic cavity and yolk sac will give rise to the notochord passing forward from Hensen's node under the ectodermal layer, the disintegration of floor of tubular notochord and the underlying endoderm is comprised by 18 th

${ }^{1}$ Department of Neurosurgery, Mersin University School of Medicine, Mersin, Turkey

${ }^{2}$ Department of Pathology, Mersin University School of Medicine, Mersin, Turkey

Received: April 24, 2018; Accepted: July 3, 2018

Online December 18, 2018

Copyright $\odot 2019$ by The Japan Neurosurgical Society

This work is licensed under a Creative Commons Attribution-

NonCommercial-NoDerivatives International License. day. As a result of this, the notochord which is now named as neuroenteric canal connects the yolk sac and the amniotic cavity through the blastopore. This temporary direct connection between the spinal cord, spine and gastrointestinal tract might result in the formation of neuroenteric cysts. ${ }^{6,7)}$ The other two theories are the splitting of the notochord as the primary event and focal endodermal-ectodermal adhesion theory respectively. ${ }^{8,9)}$

The natural history of untreated intraspinal NCs is unfavorable and the location of the cyst is the main factor of atypical clinical presentation. We describe here 16-year old boy presented with acute tetraplegia and respiratory arrest in emergency room who had cystic lesion ventral to the cervicomedullary junction.

\section{Case Presentation}

A 16-year-old boy was admitted to our hospital who have been suffering from suboccipital pain for 20 days. During this period, he had a cervical spine graph and put on analgesic therapy in another hospital. Upper extremity weakness, swallowing difficulty and urinary incontinance were the major signs of the patient just $1 \mathrm{~h}$ before admission of emergency room. He urgently underwent magnetic resonance imaging (MRI) examination in one and a half hour of admission. As soon as the patient was transported to emergency room from MRI unit than he was discovered acute tetraplegia and respiratory arrest. Immediately he was entubated and mechanically ventilated. MRI examination of the patient revealed a $1.5 \times 2.5 \times 4 \mathrm{~cm}$ well-defined intradural-extramedullar cystic heterogenous lesion extending ventral to the cervicomedullary junction. On T1-weighted images showed isointense lesion and band-shaped hyper-intensity at the posterior border of the lesion. On T2WI-weighted images exhibited mild hyper-intensity including hypo-intense area at the posterior side of cyst and displacing the cervicomedullary region to the opposite side (Figs. 1A-1D). No ischemic changes or demyelination observed on axial T2-weighted, diffusion and flair images (Figs. 1D-1F).

After consultation of patient in $45 \mathrm{~min}$, patient was referred to operation room. We urgently performed $\mathrm{C} 1$ posterior arch resection and $\mathrm{C} 2$ laminectomy in prone position. Hence the precise nature of the cyst is unknown (such as epidermoid, dermoid, hydatid cyst) after opening of the dura mater to reduce risk of aseptic chemical meningitis, the cotton chips were placed surrounding neurovascular structures and subarachnoid space to avoid spillage of the cyst contents. 

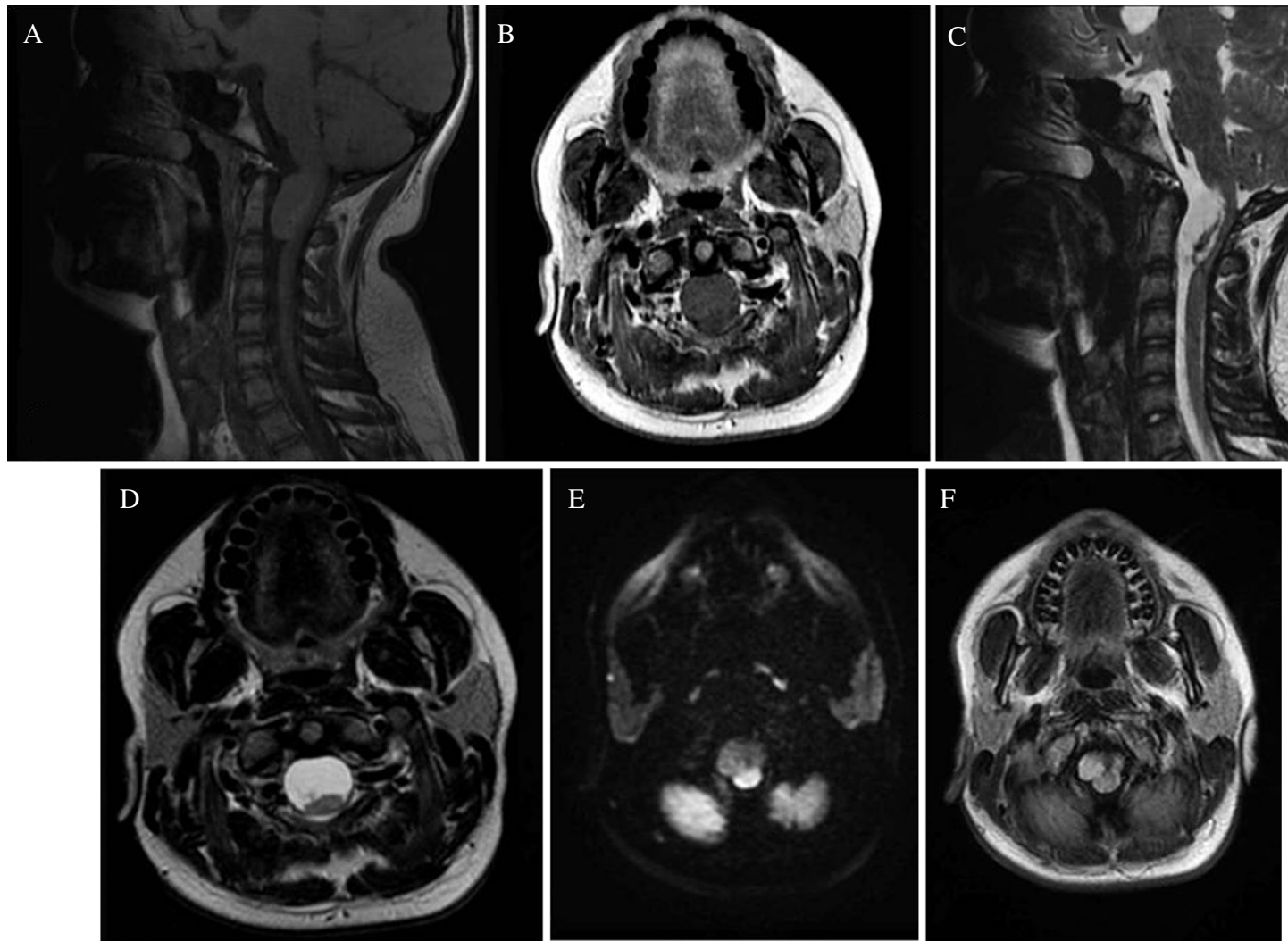

Fig. 1 T1-weighted sagittal MRI revealing an intradural cyst lying ventral to the cervicomedullary junction, displacement of this area and shows isointense lesion and band-shaped mild hyper-intensity at the lower border of the lesion (A), T1-weighted axial MRI shows severe compression of the cystic mass (B), T2-weighted sagittal image demonstrates hyper-intense cystic mass and band-shaped hypo-intensity at the posterior border of the lesion (C), axial T2-weighted scan reveals that cystic mass was filling almost cervicomedullary junction (D), diffusion-weighted imaging (E) and fluid attenuation invention recovery (F) exhibit relationship of the cyst and cervicomedullary region without any ischemic changes.

The lesion is gently punctured and whitish, viscous material aspirated slowly to avoid sudden decompression of the medulla and spinal cord. There was no feature to suggest hemorrhage. After then, the shrinked cyst wall dissected from the neural structures and subarachnoid space were totally removed. In the early post-operative period the patient has been extubated, by the time of discharge his neurological status gradually improved. He is free on mobilization by himself in the 1 st month. Follow-up MRI at 3 months showed no residual lesion (Figs. 2A-2D). Histopathologic diagnosis of the excised cyst wall was showing prominent columnar and cuboidal epithelial lining along with irregular bundles of collagen (Figs. 3A and 3B).

\section{Discussion}

Neuroenteric cysts are rare lesions of the spinal axis account for $0.7-1.3 \%$ of all spinal cord tumors. ${ }^{10)}$ These rare lesions can occur at any level of the central nervous system but are most commonly found in the lower cervical and upper thoracic segments. According to the protein/mucin content, NCs appear as avascular masses and also the signal intensity on MRI changes. On T1-weighted image; hyperintensity of the cyst can be seen in highly proteinaceous content or hemorrhage of the lesion..$^{2-5,11)}$ With contrast administrated MRI, usually no contrast enhancement is observed. However contrast enhancement pattern can be seen focally, completely or rim enhancement in rare cases. The differential diagnosis of NCs includes dermoid, epidermoid cyst, parasitic cyst, arachnoid cyst and cystic neoplasm. ${ }^{12)}$ Radiologically, it is difficult to distinguish these cystic lesions from NCs. Generally, the lack of enhancement of the cyst wall can help to differentiate NCs from cystic neoplasms. In our case, partial rim enhancement pattern is observed at the inferior border of the cystic mass. However at surgery we did not find any hemorrhage within the lesion and whitish, viscous material was inside the cystic mass which was considered for contrast enhancement.

Possible explanations for acute neurological deterioration have been proposed in NCs in the literature. The reasons of these mechanism are sudden compression with chronic distorted and compressed cervicomedullary junction, periodic accumulation of intracystic musin, dynamic factors like venous stasis with thrombosis, ball valve-like obstruction and finally hemorrhage into cyst. ${ }^{8,11,12)}$ Also it has been reported that rupture of the cyst might cause neurological exacerbation following trauma. ${ }^{7}$ Our case is unique with its fatal clinical manifestations and cervicomedullary localization of the cystic mass acute tetraplegia and respiratory arrest in the 

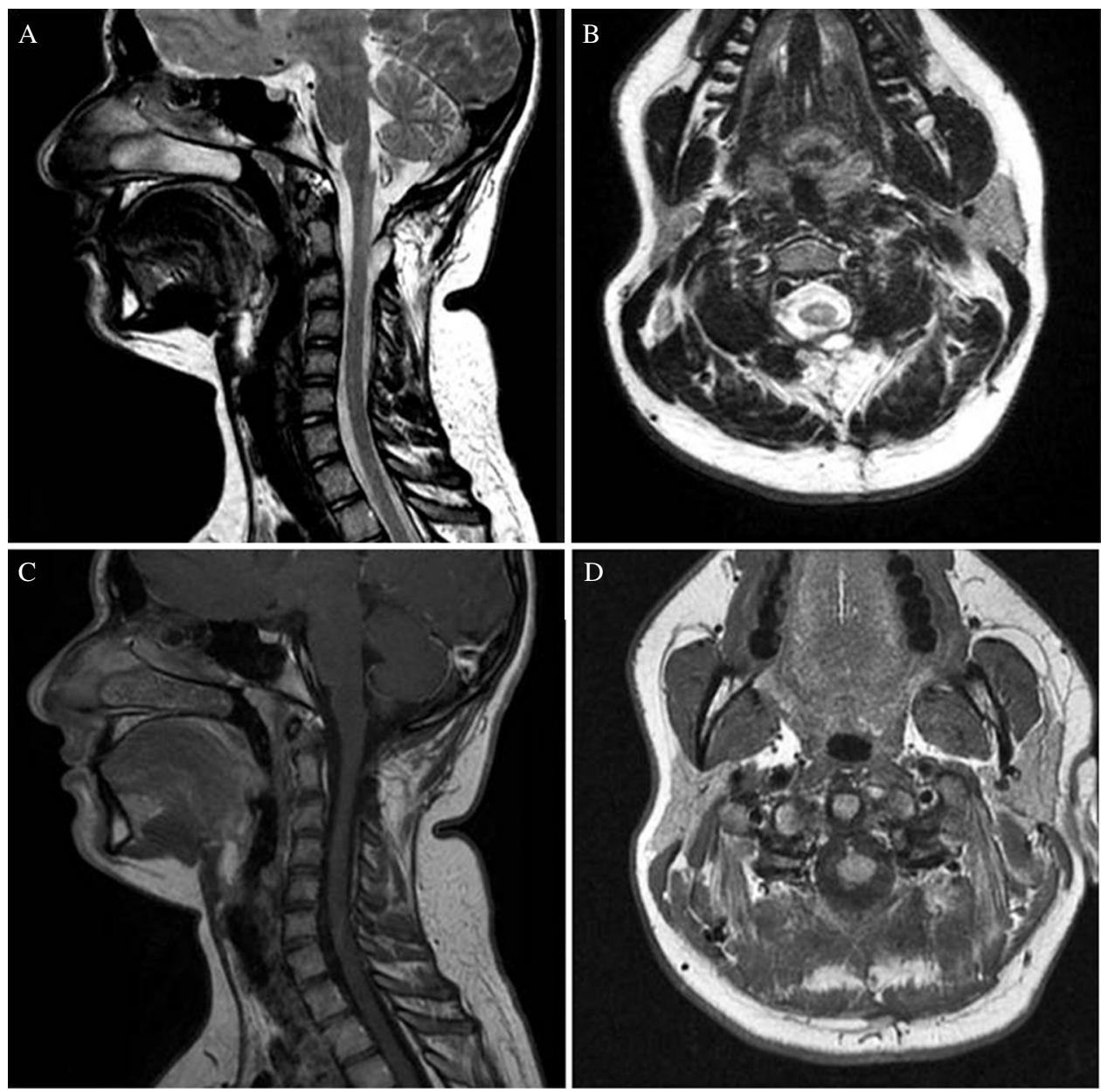

Fig. 2 The post-operative sagittal (A) and axial (B) T2 scans showing complete excision of lesion, the post-operative sagittal (C) and axial T1 (D) scans revealed decompressed cervicomedullar area.
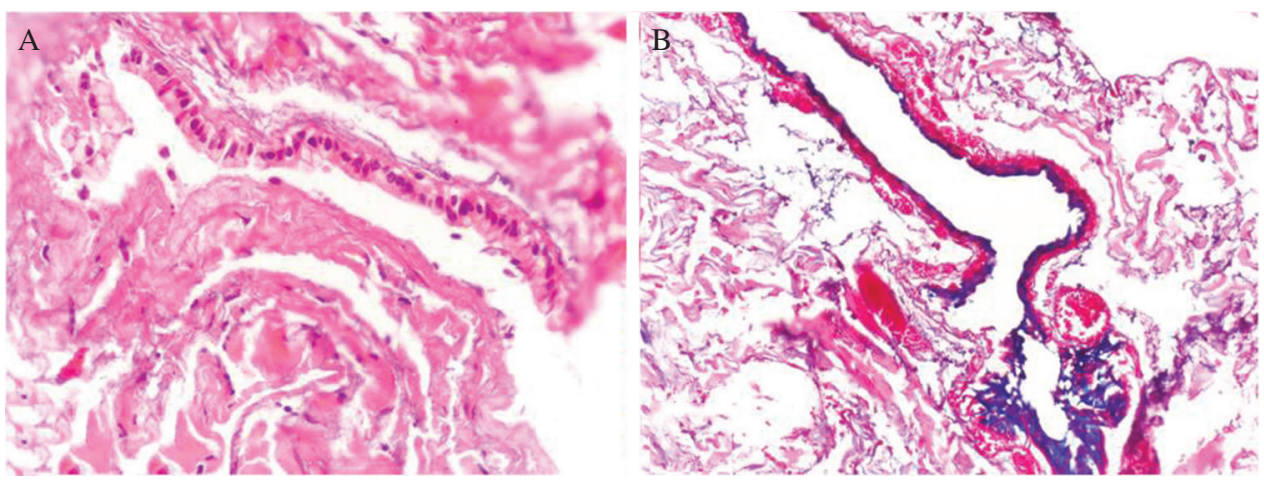

Fig. 3 Hematoxylin-eosin section photomicrographs showing prominent columnar and cuboidal epithelial lining along with irregular bundles of collagen (×200) (A), Confirmation of mucinous production can be established by histochemical assays utilizing periodic acid-Schiff $(\times 200)(B)$.

emergency room. Minor-major trauma, infection and hemorrhage in cyst are the common reasons of neurological worsening in literature; however these etiological factors were not identified in our patient. Meanwhile, the main cause of sudden catastrophic neurological deterioration is controversial. This congenital lesion is located in the anterior region of cervicomedullary junction which chronically causes distortion, stretching and compressing to neural structure. In the literature this similar mechanism was also described by Yamada et al. ${ }^{13)}$ for tethered cord syndrome. Thus, tethering of the filum terminale causes a reduction in blood flow to the spinal cord and local tissue hypoxia that eventually results dysfunction of neuronal mitochondria in the electron transport chain, which can lead to cellular dysfunction and finally death. ${ }^{13,14)}$ This pathophysiological cascade might result in serious neurological deficits with or without any trauma. Although in this case there was no known trauma, we estimate that the extended neck position could be a minor trauma leading to increased stretching or compressing 
for the underlying mechanism of sudden neurological deterioration in $35 \mathrm{~min}$ lasting cranial and cervical MRI evaluation.

It has been suggested that children with spinal cord compression might have a better prognosis for recovery in terms of neurological deficits compared to adults. ${ }^{7)}$ Although it is obviously declared by Garg et al. ${ }^{12)}$ that partial excision, location of the cyst and associated vertebral anomalies are not playing major role in the course of prognosis, duration of the follow-up has a vital importance to be considered for outcome. In the light of this data and our experience, NCs surgical outcome can be successful even though for the patients in acute onset of any vital neurological deficit and untreated delayed pediatric cases.

To the best of our knowledge, this is the first reported case of NCs associated with sudden onset of the respiratory arrest and acute tetraplegia in a child. In the analysis of patient history, simple neck pain with long-lasting latent period should be considered for advanced radiological investigation like our case. In NCs acute neurological worsening can exist without evident etiological factors.

\section{Conflicts of Interest Disclosure}

We also declare that there is no potential or actual conflicts of interest and disclosure of support related our manuscript.

\section{References}

1) Menezes AH, Traynelis VC: Spinal neurenteric cysts in the magnetic resonance imaging era. Neurosurgery 58: 97-105, 2006
2) Al-Ahmed IH, Boughamoura M, Dirks P, Kulkarni AV, Rutka JT, Drake JM: Neurosurgical management of neurenteric cysts in children. J Neurosurg Pediatr 11: 511-517, 2013

3) Gao P, Osborn AG, Smirniotopoulos JG, Palmer CA, Boyer RS: Neurenteric cysts. Pathology, imaging spectrum, and differential diagnosis. Int J Neuroradiol 1: 17-27, 1995

4) Jeon SJ, Sohn CH, Kim EH, Son KR, Park SH, Chang KH: Craniospinal neuroenteric cysts: various MR imaging features. J Korean Soc Magn Reson Med 13: 54-62, 2009

5) Savage JJ, Casey JN, McNeill IT, Sherman JH: Neurenteric cysts of the spine. J Craniovertebr Junction Spine 1: 58-63, 2010

6) Kumar R, Nayak SR: Unusual neuroenteric cysts: diagnosis and management. Pediatr Neurosurg 37: 321-330, 2002

7) Tuzun Y, Izci Y, Sengul G, Erdogan F, Suma S: Neurenteric cyst of the upper cervical spine: excision via posterior approach. Pediatr Neurosurg 42: 54-56, 2006

8) Yang T, Wu L, Fang J, Yang C, Deng X, Xu Y: Clinical presentation and surgical outcomes of intramedullary neurenteric cysts. J Neurosurg Spine 23: 99-110, 2015

9) Bremer JL: Dorsal intestinal fistula; accessory neurenteric canal; diastematomyelia. AMA Arch Pathol 54: 132-138, 1952

10) Rizk T, Lahoud GA, Maarrawi J, et al.: Acute paraplegia revealing an intraspinal neurenteric cyst in a child. Childs Nerv Syst 17: 754-757, 2001

11) de Oliveira RS, Cinalli G, Roujeau T, Sainte-Rose C, Pierre-Kahn A, Zerah M: Neurenteric cysts in children: 16 consecutive cases and review of the literature. J Neurosurg 103: 512-523, 2005

12) Garg N, Sampath S, Yasha TC, Chandramouli BA, Devi BI, Kovoor JM: Is total excision of spinal neurenteric cysts possible? Br J Neurosurg 22: 241-251, 2008

13) Yamada S, Zinke DE, Sanders D: Pathophysiology of "tethered cord syndrome." J Neurosurg 54: 494-503, 1981

14) Stetler WR, Park P, Sullivan S: Pathophysiology of adult tethered cord syndrome: review of the literature. Neurosurg Focus 29: E2, 2010 